Psychological Reports, 1991, 69, 243-251. (C) Psychological Reports 1991

\title{
THEORETICAL COMPARISON OF THE DECISION THEORIES OF J. M. KEYNES, KAHNEMAN-TVERSKY, AND EINHORN-HOGARTH ${ }^{1}$
}

MICHAEL E. BRADY

Bellflower, California
AND

\author{
California State University, Northridge
}

\begin{abstract}
Summary. - It is demonstrated that J. M. Keynes in 1921 provided a complete decision rule that handles both the cases of nonlinear probability preferences and ambiguity or uncertainty. The 1979 Kahneman-Tversky approach deals only with risk (nonlinear probability preferences) and does not specify a decision rule. The 1985-1986 Einhorn-Hogarth anchoring and adjustment rule deals only with ambiguity or uncertainty and not risk. Only Keynes' rule does both.
\end{abstract}

This paper is organized in the following way. After this brief historical introduction, we present Keynes' decision rule, Kahneman-Tversky's decision approach and Einhorn-Hogarth's decision rule. In another section, we demonstrate how Keynes' decision rule solves the Ellsberg Paradox and the Popper Paradox of Ideal Evidence quantitatively. One problem is presented for each paradox. A final section contains our conclusions.

Keynes was concerned about the following questions:

Whether ... the 'mathematical expectation' of different courses of action accurately measures what our preferences ought to be-whether, that is to say, the undesirability of a given course of action increases in direct proportion to any increase in the uncertainty of its attaining its object, or whether some allowance ought to be made for 'risk,' its undesirability increasing more than in proportion to its uncertainty (Keynes, 1921, p. 313).

Keynes then gives credit to $\mathrm{D}^{\prime}$ Alembert for being the first scholar to recognize the fact that decision makers prefer high probability values to low probability values, given equal expected monetary values (emv). Thus, if emv $v_{1}$ has a value of $\$ 90=p_{1} \cdot A_{1}$, where $p_{1}=$ the probability of success $=.9$, $\mathrm{q}_{1}=$ the probability of failure $=.1$, and $A_{1}=$ the monetary outcome equals $\$ 100$, while $\mathrm{emv}_{2}$ also has a value of $\$ 90=p_{2} \cdot A_{2}$, where $p_{2}=.1, q_{2}=.9$, and $A_{2}=\$ 900$, a large majority of decision makers will choose emv over emv $_{2}$ if probability preferences are nonlinear. Only if probability preferences are linear will the decision maker be indifferent to $\mathrm{emv}_{1}$ and $\mathrm{emv}_{2}$.

Brady and Lee (1989a, 1989b, 1989c) demonstrated that the type of problem discussed above, called by Kahneman and Tversky the "certainty effect" and by other researchers the Allais paradox or security level problem, is easily handled by Keynes' decision rule.

Unfortunately, Keynes, in his discussion of applied decision making in

\footnotetext{
${ }^{1}$ Request reprints from Dr, M. E. Brady, 9426 Flower Street, Bellflower, CA 90706 USA.
} 
the $A$ Treatise on Probability, delayed presenting his decision rule and application of it until Chapter 26 of the treatise. In his initial handling of the problem of a decision under risk and ambiguity in Chapter 6 of the treatise, there is nothing but the following brief paragraph which has been overlooked in the literature on decision making:

The conclusion, that the 'weight' and the 'probability' of an argument are independent properties, may possibly introduce a difficulty into the discussion of the application of probability to practice.' For in deciding on a course of action, it seems plausible to suppose that we ought to take account of the weight as well as the probability of different expectations (Keynes, 1921, p. 76).

The Footnote 1 in the above quote is then referenced in the following manner by Keynes: "See also Chapter XXVI. 7" (Keynes, 1921, p. 76).

Thus, to take one example, Einhorn and Hogarth (1986) state that

However, it is one thing to acknowledge the importance of ambiguity (cf. Keynes, 1921, p. 71 ...) and another to develop a Theory that incorporates it in the assessment of probabilities and the determination of choices" (p. S229).

It is clear that Keynes' important analysis in Chapter 26 of $A$ Treatise on Probability was, and has been, overlooked.

Theoretical Underpinnings of the Keynes, Kahneman-Tversky, and Einborn-Hogarth Approaches

Keynes calls his decision rule a "conventional coefficient of risk and weight." An updated description would be a "conventional coefficient of nonlinear probability preference and ambiguity." Keynes designates this coefficient as $c$. Essentially, Keynes' rule is $C \cdot A$, where $A$ is a monetary outcome. Keynes states that his rule, $C \cdot A$, is superior to the standard emv rule, $p \cdot A$. We now discuss Keynes' $c$ coefficient. Keynes states that $c$ is equal to the following:

$$
c=\frac{2 p w}{(1+q)(1+w)}, \quad 0 \leq c, w, p, q \leq 1,
$$

Now $p$ is defined as the probability of success, $q$ is defined as the probability of failure. Thus $p+q=1$. $w$ stands for weight of evidence in Keynes' formula. In a more updated description, $w$ would measure the reliability (unreliability) or ambiguity (clearness or completeness) of the information. $w$ is defined to be between 0 and 1 , just like a probability value. For instance, if $w=.1$, then the evidence or data or information is very vague, unreliable, or ambiguous. If $w=.9$, then the information or data is highly reliable or clear. If $w=0$, then one has complete ambiguity or uncertainty. A state of total ignorance exists. If $w=1$, then a situation of complete or total evidence exists, and there is no vagueness or ambiguity. 
Keynes did not believe that the situation of $w=0$ had any relevance, since we always have some information, even if slight or very slight.

The results of our endeavours are very uncertain, but we have a genuine probability, even when the evidence upon which it is founded is slight...

The difficulties which exist are not chiefly due, I think, to our ignorance of the remore future (Keynes, 1921, p. 310).

Since probability and weight are independent properties, they can be separated in Keynes' decision rule. Thus, [1] can be rewritten as

$$
c=p\left(\frac{1}{1+q}\right)\left(\frac{2 w}{1+w}\right) \text {. }
$$

Thus, $[1 /(1+q)]$ and $[2 w /(1+w)]$ are weights which allow the decision maker to take into account his probability preferences, whether they are nonlinear or linear. If his preferences are nonlinear, then he multiplies $p$ by $[1 /(1+q)]$; if his preferences are linear, then he can simply eliminate the weight $1 /(1+q)$ by multiplying by its inverse $(1+q)$, or set $q=0$. If his preferences are linear, we are left with

$$
c=p\left(\frac{2 w}{1+w}\right) .
$$

Just as the weight $[1 /(1+q)]$ dealt with probability preferences, the weight $[2 w /(1+w)]$ deals with the extent of reliability, ambiguity, or completeness of the information. If a decision maker believes his data set is completely clear and unambiguous, then he simply eliminates the reliability weight $(2 w) /(1+w)$ by multiplying by its inverse $(1+w) /(2 w)$ or by setting $w=1$.

Thus, if the decision maker's probability preferences are both linear and his information set totally complete, we are left with

$$
c=p \text {. }
$$

Thus, we see that the emv rule is a very special particular case of Keynes' generalized decision rule, which can be called a wmv (weighted monetary value). In several as yet unpublished works, Brady (1986, 1987a, $1987 \mathrm{~b}, 1988$ ) gives a similar discussion as the one above, combined with applications to economic problems as well as a complete graphical and diagrammatical analysis.

It is now time to summarize. In the terminology of Kahneman and Tversky and of Einhorn and Hogarth, Keynes' decision rule has the properties of subproportionality and subadditivity. These terms are defined below in our discussion of the other two approaches. 
Kahneman and Tversky's Prospect Theory is the result of purely experimental and empirical work. They define a weighting function, $\pi=\pi(p)$, which has, however, been given no specific form, as in the case of Keynes' $c$ coefficient. $\pi(p)$ has the following properties. First, for small values of $p, \pi$ is a subadditive function of $p$, i.e., $\pi(r p)>r \pi(p), 0<r<1$. Second, overweighting occurs for very small probabilities, starting around $1 / 1000$ or $p=.001$. Third, although $\pi(p)>P$ for very small $p$ s, for all $p$ s $0<p<1$, $\pi(p)+\pi(1-p)<1$. This property is called subcertainty. Empirically, it means the end points 0 and 1 are not defined. Finally, for a fixed ratio of $p$ s, the corresponding $\pi(p)$ is closer to 1 when the $p$ s are small than when the $p$ s are large. Thus, $\pi(p q) / \pi(p) \leq \pi(p q r) / \pi(p r)$ for $0<p, q, r<1$, where $p$, $q$, and $r$ are probabilities. This property is called subproportionality or what Keynes would call today a nonlinear probability preference for large $p$ s as opposed to small $p s$, with emv values which are identical or approximately equal. Subproportionality holds if and only if $\log \pi$ is a convex function of the $\log p$. Since subproportionality and overweighting imply subadditivity, $\pi$ must be a nonlinear function of $p$. Keynes' rule, with $w=1$, is $c=p /(1+q)$. This is a convex function and is nonlinear, although Keynes' rule is a theoretical construct and hence does not incorporate the empirical finding of overweighting. However, since such overweighting occurs at extremely small probabilities, it is doubtful that it should be incorporated in a theoretical decision rule like either the emv or wmv rules.

Kahneman and Tversky's experimental approach assumes that there is no uncertainty or ambiguity of evidence. However, they suggest a possible extension "... when the probabilities of outcomes are not explicitly given ..." (Kahneman \& Tversky, 1979, p. 288).

... decision weights may be affected by other considerations such as ambiguity or vagueness. Indeed, the work of Elsberg and Fellner implies that vagueness reduces decision weights. Consequently, subcertainty shauld be more pronounced for vague than for clear probabilities" (pp. 288-289).

At the theoretical level, Kahneman and Tversky not only have not improved on Keynes' analysis but have an inferior formulation. On the other hand, the vast amount of experimental and empirical work they have carried out serves to confirm Keynes' untested formulation, to a very large extent. From a theoretical prospective, it is subproportionality and subadditivity that are the crucial characteristics on which a decision rule needs to be generalized or based. Subcertainty and overweighting are of secondary importance. Keynes' rule $c=p /(1+q)$ satisfies the above two main properties.

Einhorn and Hogarth present an anchoring and adjustment-process model of decision making under ambiguity. We specifically concentrate, except for some general observations at the end, on that formulation of their model which deals with the case of subadditivity and ambiguity avoidance, 
as opposed to their inclusion of the case of superadditivity and ambiguity seeking. This will allow a comparison with work of Kahneman and Tversky and with Keynes concerning the standard type of problem characterized by the Allais, Ellsberg-Fellner, and Popper paradoxes. Thus, the relevant parameter values will be $0 \leq \theta \leq 1$ and $0<B<1$, where

$$
S(p)=(1-\Theta) p+\Theta\left(1-P^{B}\right), \quad 0 \leq \Theta, s(p), P, B \leq 1 .
$$

and where $S(p)$ is the final judgment resulting from the process, $\theta$ measures the amount of ambiguity, $\theta=0$ means no ambiguity so $S(p)=P$ and $\theta=1$ means complete ambiguity. Finally, $B$ is a psychological variable denoting the decision maker's attitude toward ambiguity, that is, is he seeking ambiguity or avoiding ambiguity.

$\Theta$ plays the same role in the model of Einhorn and Hogarth that $w$ plays in Keynes' wmv model, except that the endpoints express opposite conditions. Thus, if there is no ambiguity in Keynes' model, $w=1$ as opposed to $\Theta=0$ in the model of Einhorn and Hogarth. Similarly, in Keynes' model, if $w=0$, there is complete ambiguity. This is designated as $\theta=1$ by Einhorn and Hogarth.

The latter state that their "version of the ambiguity model ... bears a striking resemblance to the decision-weight function of Prospect Theory. In that theory, the effects of uncertainty on choice are modeled via a decision weight function, $\pi(p)$, that is subadditive, has undefined endpoints (subcertainty) and displays subproportionality. This latter characteristic implies that the slope of the decision weight function is less than one and decreases as $p$ gets smaller" (Einhorn \& Hogarth, 1986, p. S235).

Thus, the theory of Einhorn and Hogarth, which deals only with ambiguity and not with the case of well-defined probabilities and risk à la Kahneman and Tversky, also results in subadditivity, subproportionality, and overweighting, though not undefined endpoints.

Einhorn and Hogarth then present a decision rule which they call "expected worth under ambiguity" (EWA), where

$$
E W A=W_{\mathrm{G}} S\left(p_{\mathrm{G}}\right)+w_{\mathrm{L}} S\left(P_{\mathrm{L}}\right),
$$

where $w_{\mathrm{G}}$ and $w_{\mathrm{L}}$ are the subjective worths of the amounts to be gained and lost in a two-outcome gamble, and $S\left(p_{\mathrm{G}}\right)$ and $S\left(P_{\mathrm{L}}\right)$ are the ambiguous probabilities of gaining and losing. The goal is to maximize EWA.

If $\Theta=0$, then $S\left(p_{\mathrm{G}}\right)=p_{\mathrm{G}}$ and $S\left(P_{\mathrm{L}}\right)=P_{\mathrm{L}}$ and the model of Einhorn and Hogarth is equivalent to the usual subjective model of expected utility. Although Keynes' rule does not incorporate the utility of monetary outcomes, but only the monetary outcome itself, i.e., $A$ instead of $\mathrm{U}(\mathrm{A})$, it is straightforward to make this substitution in Keynes' decision rule. 
Einhorn and Hogarth (1986) indirectly admit that they have overlooked Keynes' decision rule:

We are aware of only two other rules for dealing with ambiguous choice; one propounded by Ellsberg . . . and the other by Gärdenfors and Sahlin. However, neither of these rules allows for ambiguity seeking and avoidance (p. S244).

Keynes' rule allows for ambiguity avoidance but not for ambiguity seek. ing. As discussed by Brady and Lee (1989b), Keynes' rule does incorporate a "reflection effect." Under risk, given certain gains versus only probable gains, the decision maker is risk averse; given certain losses versus only prob. able losses, the decision maker, in attempting to avoid the certain loss, "appears" to be a risk taker. The same type of reflection effect occurs in Keynes' rule under ambiguity.

Finally, Keynes' rule explicitly deals with both risk and ambiguity; the EWA rule of Einhorn and Hogarth deals only with ambiguity.

A final comment deals with the issue of whether the size of the probabilities used to exemplify ambiguity seeking in the model of Einhorn and Hogarth is relevant. Probabilities of $.001(1 / 1000)$ should be designated as extremely low, not merely as low. "Low" connotes probabilities in the .01 to .33 range, "medium" is associated with probabilities in the .34 to .67 range, and "high" is generally associated with probabilities in the .68 to .99 range. Thus, while .5 would certainly be classified as a medium probability and is the probability chosen to exemplify avoidance of ambiguity in discussing gains by Einhorn and Hogarth, .001 is not a low probability, which is the probability used by Einhorn and Hogarth when discussing ambiguity seeking under gains.

\section{Quantitative Solutions to the Ellsberg and Popper Paradoxes}

Consider the Ellsberg Paradox first (see Ellsberg, 1961, pp. 650-654). The decision maker is presented the choice of choosing between two different urns (U) on which to bet, U I and U II. U I has 100 balls in it, of which 50 are red and 50 are black. U II also has 100 balls in it; however, in this urn, the number of black balls can range from 0 to 100 and the number of red balls can range from 100 to 0 . If one draws a black ball, one receives $A=\$ 100$. If one draws a red ball, one receives $A=\$ 0$.

Now the probabilities of $U I$ are $p_{\mathrm{B}}=1 / 2$ and $p_{\mathrm{R}}=1 / 2$, where $p$ denotes probability, $\mathrm{B}$ denotes black, and $\mathrm{R}$ denotes red. The information set is total and complete. No additional information could possibly lead to a revision of $p_{\mathrm{R}}$ and $p_{\mathrm{B}}$. According to Keynes, $w=1$, while according to Einhorn and Hogarth, $\theta=0$. Further, the probabilities are objective.

To compute probabilities for U II, one must resort to the principle of indifference. Since we are dealing with basic indivisibilities, one can assign a subjective a priori probability of $p_{\mathrm{B}}=1 / 2$ and $p_{\mathrm{R}}=1 / 2$. However, the confi- 
dence or reliability a decision maker feels in his estimation of the probabilities of U II balls is quite substantially less than the confidence associated with U I ball estimates. Essentially, U I is a problem of risk, while U II is a problem of uncertainty. For purposes of exposition, assign a $w$ to U II of, say, 0.35 . Let us compute the wmvs for U I and U II. Since this is a problem of ambiguity, multiply by $(1+q)$ to remove the risk weight. For U I, the wmv is

$$
\begin{aligned}
p_{\mathrm{B}} & \left(\frac{2 w}{1+w}\right) A+p_{\mathrm{R}}\left(\frac{2 w}{1+w}\right) A \\
& =1 / 2 \cdot(1) \cdot \$ 100+1 / 2 \cdot(1) \cdot \$ 0 \\
& =\$ 50 .
\end{aligned}
$$

For U II, the wmv is

$$
\begin{aligned}
p_{\mathrm{B}} & \left(\frac{2 w}{1+w}\right) A+p_{\mathrm{R}}\left(\frac{2 w}{1+w}\right) A \\
& =1 / 2 \cdot(0.35) \cdot 100+1 / 2 \cdot(0.35) \cdot \$ 0 \\
& =\$ 17.50 .
\end{aligned}
$$

Thus, U I is preferred to U II as a bet. According to followers of subjective expected utility such as Savage, this is paradoxical since the decision maker should be indifferent. It should also be mentioned that in a variate of this problem, Ellsberg (1961) allowed decision makers to draw out a two- or four-ball sample from U II. There was no appreciable change in the choices made. A majority of decision makers chose to bet on U I. Thus, decision makers are ambiguity averse, just as they are risk averse.

We now turn to the Popper paradox (see Popper, 1959, pp. 406-418). Suppose one is asked to estimate the probability of drawing a "spade" card from a deck of cards. One's subjective a priori estimate would be $1 / 4$. Now suppose one draws a card one million times with replacement. One finds that the probability of drawing a "spade" is $1 / 4$. Let us designate $b$ to stand for the hypothesis that "one will draw a spade." Let $e$ stand for the experimental evidence involved in drawing from this deck. The marginal a priori estimate, $p(b)$, was $1 / 4$. The conditional calculation, based on experimental evidence, $p(b / e)$, was also $1 / 4$. We get

$$
p(b)=1 / 4=p(b / e) .
$$

According to Popper, this means that for subjective probability proponents, experimental and/or empirical evidence is, in effect, irrelevant. Popper 
then argues for a relative (or limiting) frequency interpretation for probability.

Again, Popper's paradox is easily handled by Keynes' decision rule. Obviously, for $p(b / e), w=1.0$. This means that the information set is complete. However, just as obviously, the $w$ for $p(b)$, our a priori estimate made with no experimental evidence (i.e., only specificatory evidence regarding questions like, "Is this a fair deck?", "Are any of the cards bent or otherwise marked?", etc.) $=0$.

Let us suppose that if one draws a spade, one gets $\$ 50$ and if one draws any other kind, one gets $\$ 0$. Then for our a priori estimate, we obtain the following. The

$$
\begin{aligned}
w m v & =p\left(\frac{2 w}{1+w}\right) A \\
& =1 / 4 \cdot(0) \cdot \$ 50+3 / 4 \cdot(0) \cdot \$ 0 \\
& =\$ 0.00 .
\end{aligned}
$$

For our case where we have made one million draws with replacement, we get the following. The

$$
\begin{aligned}
\text { wmv } & =p\left(\frac{2 w}{1+w}\right) A \\
& =1 / 4 \cdot(1) \cdot \$ 50+3 / 4 \cdot(1) \cdot \$ 0 \\
& =\$ 12.50 .
\end{aligned}
$$

What Popper did not realize was that the confidence or the reliability of probabilities calculated a priori with no experimental or empirical evidence is nil while the confidence in probabilities based on large amounts of data is extremely high. Once Keynes' concept of weight of evidence, $w$, is made explicit, Popper's attack on subjective probability fails.

In the opinion of the authors, Keynes' decision rule, his conventional coefficient of risk and weight, which was first presented by Keynes in 1908 in his successful fellowship dissertation at Cambridge and formally published in Chapter 26 of A Treatise on Probability in 1921, is at least equal in explanatory power, simplicity of use, and generality to any other "modern" decision rule or approach, be it Kahneman and Tversky's or Einhorn and Hogarth's.

\section{REFERENCES}

Brady, M. E. (1986) On the application of Keynes's logical probabjlity approach. (Unpublished manuscript)

Brady, M. E. (1987a) J. M. Keynes's criteria for decision making under uncertainty and its relation to Prospect Theory. (Unpublished manuscript) 
Brady, M. E. (1987b) Uncertainty in Keynes's A Treatise on Probability and the General Theo$r y$. (Unpublished manuscript)

Brady, M. E. (1988) J. M. Keynes's logical Bayesian, weighted monetary value approach to decision making under conditions of risk and uncertainty. (Unpublished manuscript)

BRADY, M. E., \& LEE, H. B. (1989a) Dynamics of choice behavior: the logical reaction between linear objective probability and nonlinear subjective probability. Psychological Reports, 64, 91-97.

Brady, M. E., \& LEE, H. B. (1989b) Is there an Ellsberg-Fellner paradox? A note on its resolution. Psychological Reports, 64, 1087-1090.

BRADY, M. E., \& LEE, H. B. (1989c) Is there an Allais paradox? A note on its resolution. Psychological Reports, 64, 1223-1230.

EInHoRn, H. J., \& Hogarth, R. M. (1985) Ambiguity and uncertainty in probabilistic inference. Psycbological Review, 92, 433-461.

Enhorn, H. J., \& Hogarth, R. M. (1986) Decision making under ambiguity. Journal of Business, 59, S225-S250.

Ellsberg, D. (1961) Risk, ambiguity, and the Savage axioms. Quarterly Journal of Economics, $75,643.669$.

Kahneman, D., \& Tversky, A. (1979) Prospect Theory: an analysis of decision under risk. Econometrica, 47, 263-291.

Keynes, J. M. (1921) A treatise on probability. London: Macmillan. (AMS Press Reprint, 1979)

Popper, K. (1959) The logic of scientific discovery. London: Routledge \& Kegan-Paul.

Accepted August 1, 1991. 